\title{
Blasses oder Bleiches Knabenkraut (Orchis pallens)
}

\author{
Kurt Baumann
}

\begin{abstract}
Pale Orchid (Orchis pallens) is orchid of the year 2012. Its biology, ecology and distribution are summarized. In addition some similar orchids with pale yellow flowers are mentioned.
\end{abstract}

\section{Zusammenfassung}

Das Blasse Knabenkraut (Orchis pallens) ist Orchidee des Jahres 2012. Biologie, Ökologie und Verbreitung werden vorgestellt. Außerdem werden ähnliche blassgelbe Orchideen erwähnt.

\section{Einführung}

Die deutschen Namen der (seit 1989) 24. Orchidee des Jahres entsprechen der wissenschaftlichen Artbezeichnung, die man mit blass, bleich oder auch gelblich übersetzen kann. Von beiden geläufigen Bezeichnungen konnte sich bisher keine durchsetzen, so dass häufig beide angeführt werden. Beide beziehen sich auf die Blütenfarbe, die keinerlei Spur von Rot enthält, wie auch das ungültige Synonym Orchis sulphurea (= schwefelgelb) ausdrückt.

Die Art wurde zum ersten Mal von Cordus im Jahr 1543 in einem Werk erwähnt, das GEsNER erst posthum 1561 veröffentlichte (KüNKELE \& Baumann 1998). Die älteste Abbildung, nach einer Vorzeichnung von LEONHART Fuchs wahrscheinlich von Füllmaurer zwischen 1543 und 1557 gemalt und von SPECKLIN

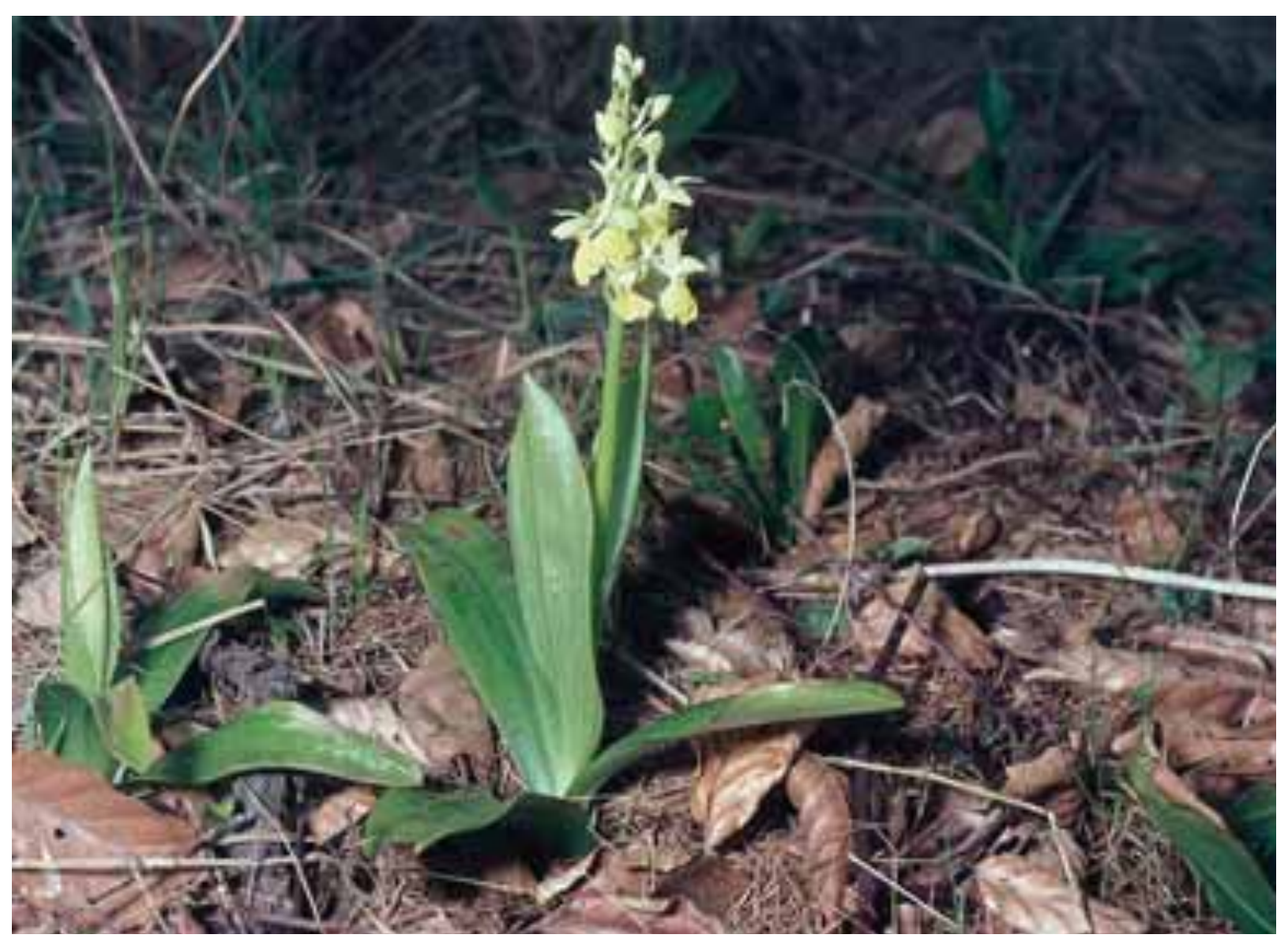


in Holz geschnitten, findet sich im nicht gedruckten Manuskript zur 2. Auflage des „New Kreüterbuchs“ von Fuchs. Die Druckstöcke der Holzschnitte wurden von BAUHin 1651 für sein Pflanzenwerk verwendet (SeYbold 1986). Beide Angaben (Text und Abbildung) beziehen sich auf Funde von der Schwäbischen Alb. Fuchs bezeichnet die Pflanze als Orchis lutea und Geel Knabenkraut.

\section{Bau der Pflanze}

Das Blasse Knabenkraut ist wie viele andere Erdorchideen unserer Heimat ein Knollengeophyt, d. h. die ungünstige, kalte Winterjahreszeit wird unterhalb der Erdoberfläche in Knollenform überstanden (andere Möglichkeiten wären Zwiebel oder Wurzelstock). Die Knollen sind längliche, eiförmige Gebilde ohne fingerförmige Verlängerungen, wie sie für die Knollen der Orchideen der Gattung Dactylorhiza (das ebenfalls Knabenkraut oder neuerdings auch Fingerwurz genannt wird) typisch sind. Die Wurzeln entstehen an der Basis des sich bildenden Stängels. Zur Blütezeit liegen zwei Knollen vor, die vorjährige, aus der der diesjährige Blütentrieb gewachsen ist, und eine junge, gestielte aus einer Niederblattachsel (Füller 1962). Während der sommerlichen Trockenheit verfällt die ältere Knolle und stirbt ab. Die jüngere trägt an der Spitze eine nicht gut erkennbare Knospe (Vöтн 1999), aus der dann im Herbst und Frühwinter ein Spross bis zur Erdoberfläche wächst. Im Frühjahr manchmal auch schon Ende Februar (BAUMANN 2005) - treiben die Laubblätter aus. Sind Knolle und Blätter kräftig genug, kommt es zu einem Blütentrieb, der nach der Fruchtreife abstirbt.

Der kräftige Blütenspross wird 15-30 (40) cm hoch (Reinhardt 1991, Presser 2002). Am Grunde trägt er 2-3 Schuppenblätter. Von den 4-6 hellgrünen, ungefleckten Laubblättern sind

Abb. 1 (Seite 46): Orchis pallens, Übersicht.

Abb. 2 (oben): Orchis pallens.

Abb. 3 (unten): Detail der Blüte von Orchis pallens.
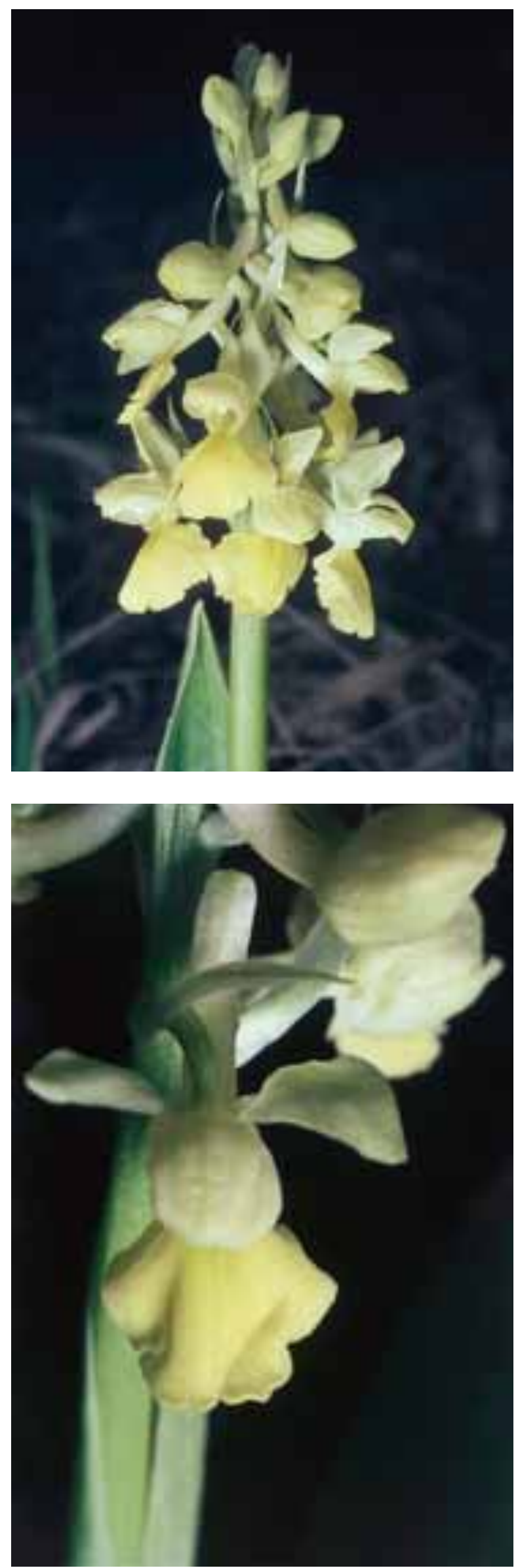

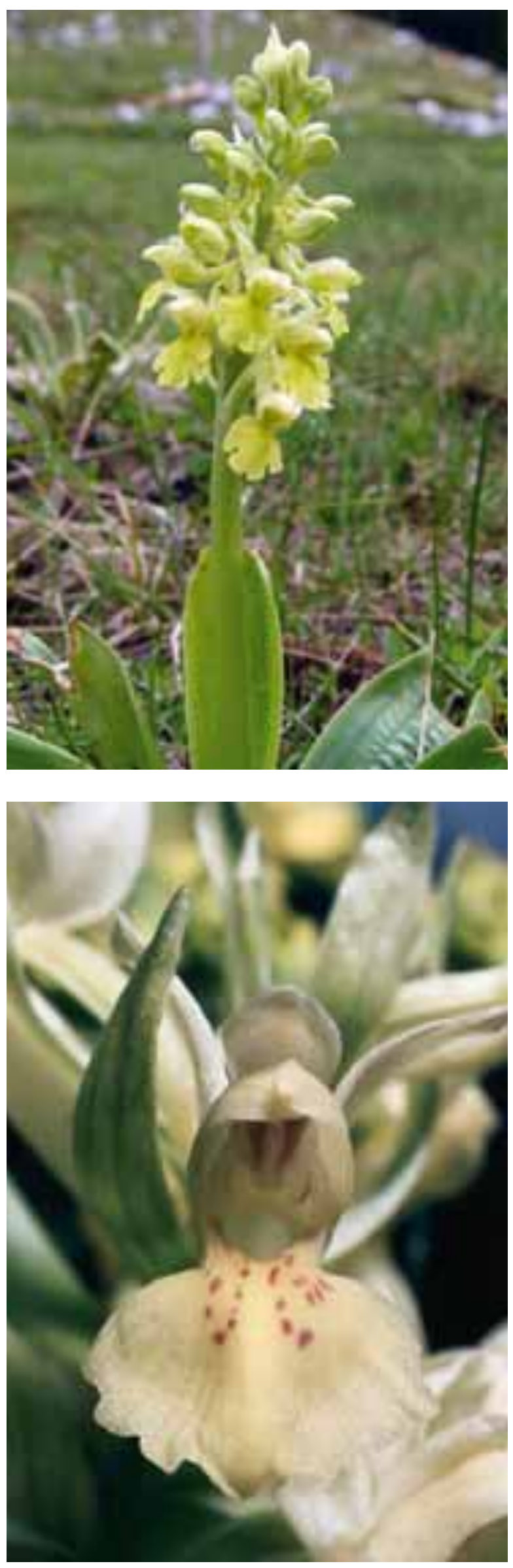

$2-4$ rosettig angeordnet. Sie sind meist $8-15 \mathrm{~cm}$ lang und $2-5 \mathrm{~cm}$ breit, Größenangaben von Buttler (1986) liegen bei einer Länge von $6-12 \mathrm{~cm}$ und einer Breite von 1,5-4 cm. Sie glänzen vor allem auf ihrer Unterseite. Am Ende sind sie schwach zugespitzt (KretzSCHMar 2008). Noch in der unteren Stängelhälfte folgen 1-2 schmalere Blätter, die dem Stängel fest anliegen und ihn kurz scheidig umfassen. Sie erreichen den Blütenstand nicht, so dass der Stängel frei liegt.

\section{Blüte und Frucht}

Das Bleiche Knabenkraut ist eine der am frühesten blühenden einheimischen Orchideen. Die Hauptblütezeit liegt zwischen Ende April und Mitte Mai, aber bereits Anfang April kann man blühende Exemplare finden. Deshalb wird sie durch späte Schneefälle und Fröste geschädigt, da der Blütenstand nicht mehr durch eine Laubdecke geschützt ist (Vӧтн 1999), und setzt auch öfters mit der Blüte aus. Im Gebirge blüht sie noch bis Mitte Juni.

Der allseitswendige, breit zylindrische Blütenstand wird 3,5-9 cm lang und ist mit 6-35 Blüten besetzt. Die Hochblätter, aus deren Achsel die Blüten entspringen, sind grünlich bis hellgelblich, häutig-durchscheinend und nicht krautig wie bei der Gattung Fingerwurz. Dies ist neben dem Knollenbau ein weiteres Unterscheidungsmerkmal zwischen den Gattungen Orchis und Dactylorhiza. Die Hochblätter sind mit 15-21 mm ungefähr so lang wie oder etwas länger als der grüne, gedrehte Fruchtknoten.

Die Blüten sollen vor allem am Abend wie Schwarzer Holunder duften (Presser 2002), wobei der Duft als intensiv und unangenehm beschrieben wird. Von manchen wird er wie Katzenharn empfunden (Reinhard 1991).

Der Bau der Blüte entspricht dem Bauplan der typischen Orchideenblüte und umfasst zwei

Abb. 4 (oben): Orchis pallens am Olymp in Griechenland. Abb. 5 (unten): Blüte der gelben Form von Dactylorhiza sambucina.

Abb. 6 (Seite 49): Ausschnitt aus dem Blütenstand von Dactylorhiza incarnata. 
Blütenblattkreise mit je drei Blütenblättern. Die Blüte des Blassen Knabenkrauts gehört wie die Blüte des Manns-Knabenkrautes zu den „Flügelorchis“-Typen (FüLLER 1962), bei denen die beiden seitlichen Blütenblätter des äußeren Kreises aufgerichtet und meist rückwärts nach außen gedreht sind. Sie sind 8-11 mm lang, 4-6 $\mathrm{mm}$ breit und von schief eiförmiger Gestalt. Das mittlere Sepalum ist symmetrisch eiförmig, aber nur 7-9 mm lang. Es bedeckt mit den beiden asymmetrischen, 7-8 $\mathrm{mm}$ langen Petalen das Säulchen, den Geschlechtsapparat der Orchideenblüte. Nach Baumann \& KüNKele (1988) sind die Petalen nur etwa 5-6 mm lang. Das mittlere nach unten gerichtete Blütenblatt des inneren Kreises ist die Lippe, der Hauptschauapparat und Landeplatz für bestäubende Insekten. Die Lippe ist seicht dreilappig geformt mit schwach geteiltem Mittellappen. Die Seitenlappen sind abgerundet und ganzrandig. Im zentralen Teil ist sie dunkler gefärbt, aber sonst ebenso hellgelb wie die anderen Blütenblätter und ohne jegliche Zeichnung. Sie ist fast flach und breiter als lang, nach REINHARD (1991) 6-11 mm lang und 7-14 mm breit. Der zylindrische Sporn ist meist aufwärts gebogen, am Ende stumpf und ungefähr ebenso lang wie der Fruchtknoten. Das kurze Säulchen ist hellgelb gefärbt wie die lang gestielten Pollenpakete, die mit ihren getrennten Klebscheiben in einem gemeinsamen Beutelchen direkt über der herzförmigen Narbenhöhle stehen. Versucht ein Insekt mit seinem Rüssel den Nektar aus dem Sporn zu saugen, zerreißt es bei unvermeidbarer Berührung das empfindliche Beutelchen, und die Klebscheiben heften sich mit dem Klebstoff am Rüssel oder dem Kopf des Insekts fest. Die Pollenpakete werden beim $\mathrm{Zu}$ rückziehen des Kopfes aus ihren Fächern gerissen. Die zuerst nach oben gerichteten Stielchen knicken in sehr kurzer Zeit nach vorn um, so dass beim nächsten Blütenbesuch das Pollenpaket oder Teile davon auf der klebrigen Narbe landen (FüLLER 1961).

Als Bestäuber treten bei den früh blühenden Orchideen-Arten Blasses Knabenkraut und Holunder-Knabenkraut (Dactylorhiza sambucina) überwiegend Hummelköniginnen der ein-

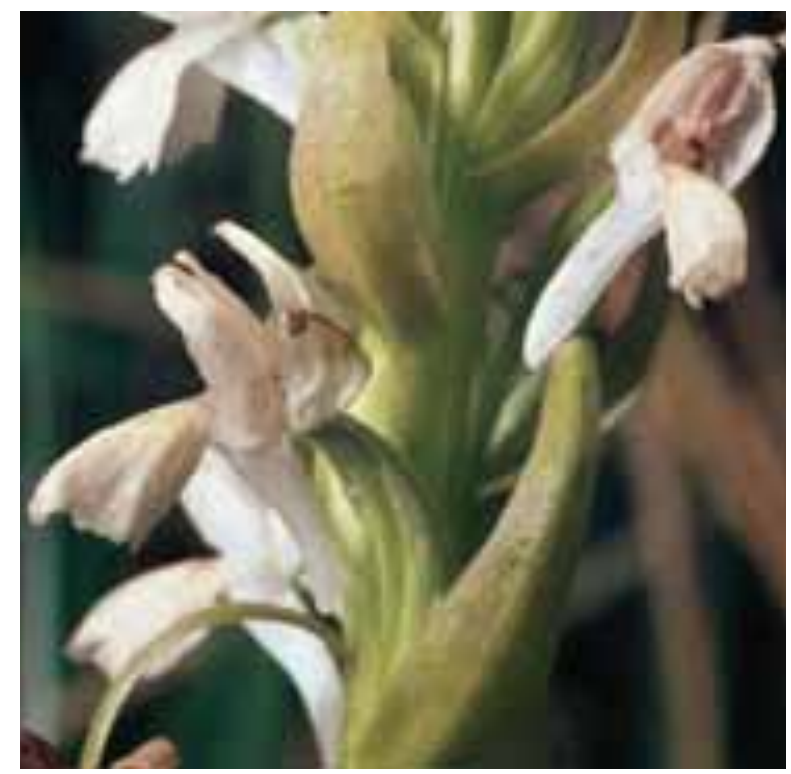

heimischen Arten Bombus hortorum (Gartenhummel), B. lapidarius (Steinhummel) und B. pascuorum (Feldhummel) auf (Vöтн 1999, Baumann 2005). Sie sollen dabei von der Frühlingsplatterbse auf das Blasse Knabenkraut überwechseln. Dass es sich beim Blassen Knabenkraut um Fremdbestäubung durch Insekten handelt, ist daran festzustellen, dass der Bestäubungserfolg im unteren Drittel des Blütenstandes größer ist, was bei Selbstbestäubung nicht der Fall wäre. Auch sind die Kapseln im Fruchtstand unregelmäßig verteilt. Während bei BAUMANN (2005) von einem Fruchtansatz von 54-59\% berichtet wird, gibt Vöтн (1999) in seinem Gebiet (Niederösterreich) nur 13\% an.

Die Kapselfrucht ist 12-24 mm lang und 5-6 mm dick. Die Samen sind wie bei allen Orchideen sehr klein, da sie keine Nährstoffe enthalten. Für Orchis pallens werden eine Länge von $0,35-0,51 \mathrm{~mm}$ und eine Breite von 0,14$0,2 \mathrm{~mm}$ angegeben. Der Embryo ist in dem winzigen Samen von einer blasenförmigen Hülle aus großen verkorkten Zellen umgeben. Sie ist schwer benetzbar und erleichtert das Einspülen in tiefere Erdschichten. Die Hülle macht den Samen sehr leicht, so dass er als Blasenflieger vom Wind ausgebreitet werden kann.

Die Keimung und Entwicklung der Pflanze ist nicht ohne die Hilfe eines Pilzes möglich, der ihr die notwendigen Nährstoffe liefert. Da von einer Gegenleistung der Orchidee nichts 


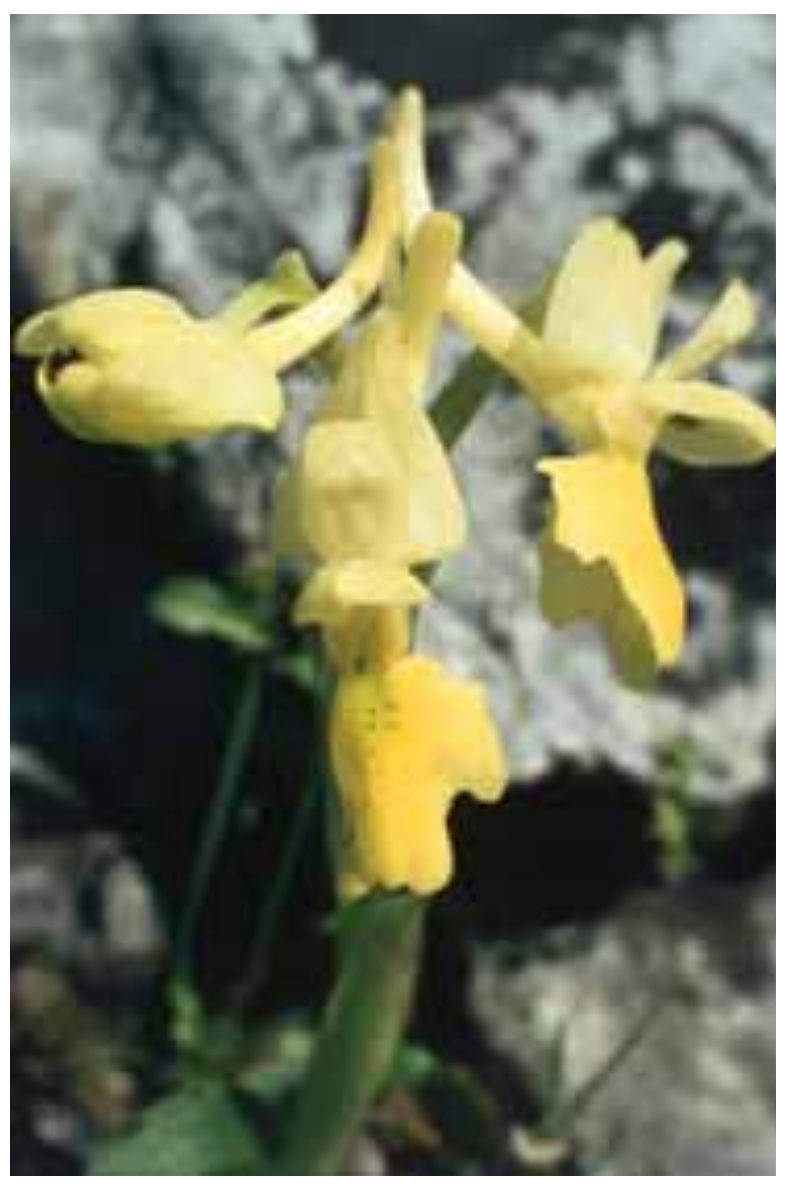

bekannt ist, handelt es sich also um Parasitismus. Erst im Besitz grüner Blätter kann die Pflanze assimilieren und Nährstoffe in ihrer Knolle anreichern. Nach einer Zeichnung von Ziegenspeck (abgebildet in Füller 1961) liegt die erste Blattbildung erst im 6. Sommer vor, nach BaUmann (2005) dauert es nicht so lang.

\section{4. Ökologie und Verbreitung}

Das Blasse Knabenkraut ist eine Halbschattenpflanze, ein Mäßigwärme- und Frischezeiger, wächst von schwach sauren bis stark basischen Böden und auf stickstoffarmen bis mäßig stickstoffreichen Standorten. Diese Orchidee bevorzugt frische, neutrale, basenreiche (kalkhaltige), milde, humose, mittel- bis tiefgründige, lockere Lehm- und Tonböden (BAumanN 2005). Sie wächst an Waldrändern, an grasigen, hellen Stellen in Buchen- und Eichenwäldern, in sonnigen Schluchtwäldern, seltener in $\mathrm{Na}$ delwäldern. Sie steht auch in buschreichem Gelände auf ehemaligen Kuhweiden und gern in Gebieten mit Niederwaldwirtschaft (Presser
2002). Während sie in niederen Lagen an schattigen Standorten vorkommt, steht sie in höheren Lagen im Mittelgebirge und in den Alpen sogar sonnig an Südhängen in Bergwiesen. Sie kommt von $150 \mathrm{~m}$ bis $1600 \mathrm{~m}$ (selten über $2000 \mathrm{~m}$ ) vor (Aeschimann et al. 2004), in Griechenland und der Türkei soll sie bis $2400 \mathrm{~m}$ hoch steigen.

Das Bleiche Knabenkraut ist eine submediterrane und montane Art (Eberle 1982). Seine Verbreitung erstreckt sich über die Gebirge Europas und Vorderasiens, von Nordspanien über SW- und Südfrankreich, Süd- und Mitteldeutschland, Schweiz, Italien, Griechenland, Türkei bis Transkaukasien. Es kommt auf der Krim vor, zudem in Rumänien, Ungarn, Tschechien und Südpolen (Baumann 2006). Es fehlt völlig im atlantischen Europa und im Norden. Die Nordwestgrenze der Verbreitung verläuft durch Mitteldeutschland. In Deutschland haben die Vorkommen zwei klare Schwerpunkte, zum einen Nordost-Hessen und Thüringen, zum anderen die Schwäbische Alb. In 11 Bundesländern fehlt die Orchidee. Orchis pallens zeigt wie viele andere Orchideen einen Rückgang. Gründe sind zu starke Beschattung der Standorte auf Grund der fehlenden Walddynamik, Aufgabe der Niederwaldwirtschaft, Verbiss durch zu große Wildbestände und Ausgraben der Knollen durch Wildschweine (nicht durch Menschen - die medizinische Verwendung der Knollen gegen Husten bei Kindern ist schon lange vorbei). Als einziges Bundesland meldet Hessen seine Bestände als nicht gefährdet, Baden-Württemberg und Sachsen-Anhalt dagegen als gefährdet, Thüringen und Bayern als stark gefährdet.

\section{Verwandte und}

\section{Verwechslungsmöglichkeiten}

Das Blasse Knabenkraut zeigt kaum Variabilität. Es wird deshalb auch nicht in Unterarten aufgegliedert. Es wird u.a. mit dem Manns-

\section{Abb. 7 (oben): Orchis pauciflora.}

Abb. 8 (Seite 51): Orchis pauciflora in einem Massenbestand in den Abruzzen. 
Knabenkraut und der Spitzels-Orchis zu einer Sektion oder Untergattung zusammengefasst. Hybriden werden nur mit den Arten dieser Sektion gebildet, nicht aber mit anderen OrchisArten. Aus Deutschland ist der Bastard mit dem Manns-Knabenkraut unter dem Namen Orchis x hausknechtii seit 1885 bekannt. Wenn auf den Blüten rötliche Farbtöne auftreten, weist dies auf eine Hybridisierung hin. Aus Frankreich und der Schweiz werden auch Bastarde mit Orchis spitzelii gemeldet (in Deutschland ist diese Art ausgestorben). Ihr Name ist Orchis x klopfensteiniae. Sehr fragwürdig und unwahrscheinlich sind die früher gemeldeten Hybriden mit dem Holunder-Knabenkraut.

Eigentlich ist das Blasse Knabenkraut wegen seiner frühen Blütezeit und der glänzenden Blätter nicht mit anderen Arten zu verwechseln. Aber es gibt noch eine Anzahl gelbblühender Orchideen, die Orchis pallens ähneln.

In Deutschland sind dies Dactylorhiza ochroleuca und Dactylorhiza sambucina. Erstere Art wird von manchen als Unterart des Fleischfar- benen Knabenkrautes (Dactylorhiza incarnata) angesehen. Sie hat blassgelbe Blüten ohne Zeichnung, die Blätter stehen aber eng am Stängel und sie blüht ab Ende Mai auf nassen, kalkhaltigen Böden. Der Sporn ist nach unten gerichtet wie auch beim Holunder-Knabenkraut. Das Holunder-Knabenkraut hat fast immer rote Punkte auf der gezähnten Lippe, deren Mittellappen spitz ausläuft. Sie blüht schon Anfang Mai. In den Beständen treten meist auch rotblütige Pflanzen auf (Presser 2002).

Im Mittelmeerraum von Nordspanien, Südfrankreich, Italien, Dalmatien, Griechenland bis zur Türkei kommt Orchis provincialis (Provence-Knabenkraut) vor. Es hat gelbe Blüten mit rot gepunkteter, ganzrandiger Lippe. Die Lippe ist schafsnasenartig gewölbt, der Sporn nach oben gebogen. Die in Rosetten angeordneten Blätter sind mit purpurnen Flecken gezeichnet. Orchis pauciflora (Wenigblütiges Knabenkraut) tritt in Korsika, in Sizilien, auf dem Apennin, an der dalmatischen Küste und in Griechenland auf. Die gelben Blüten sind im

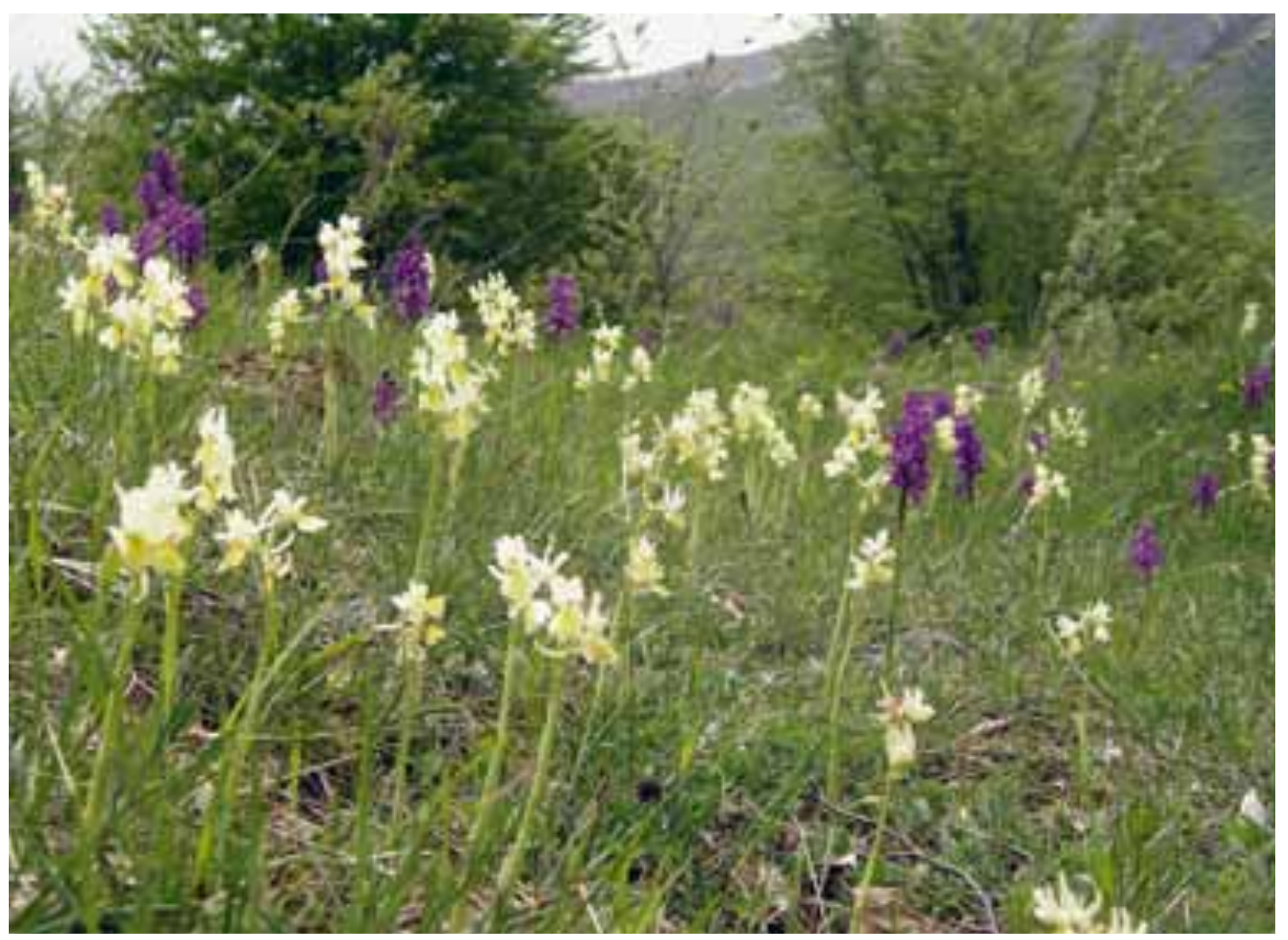



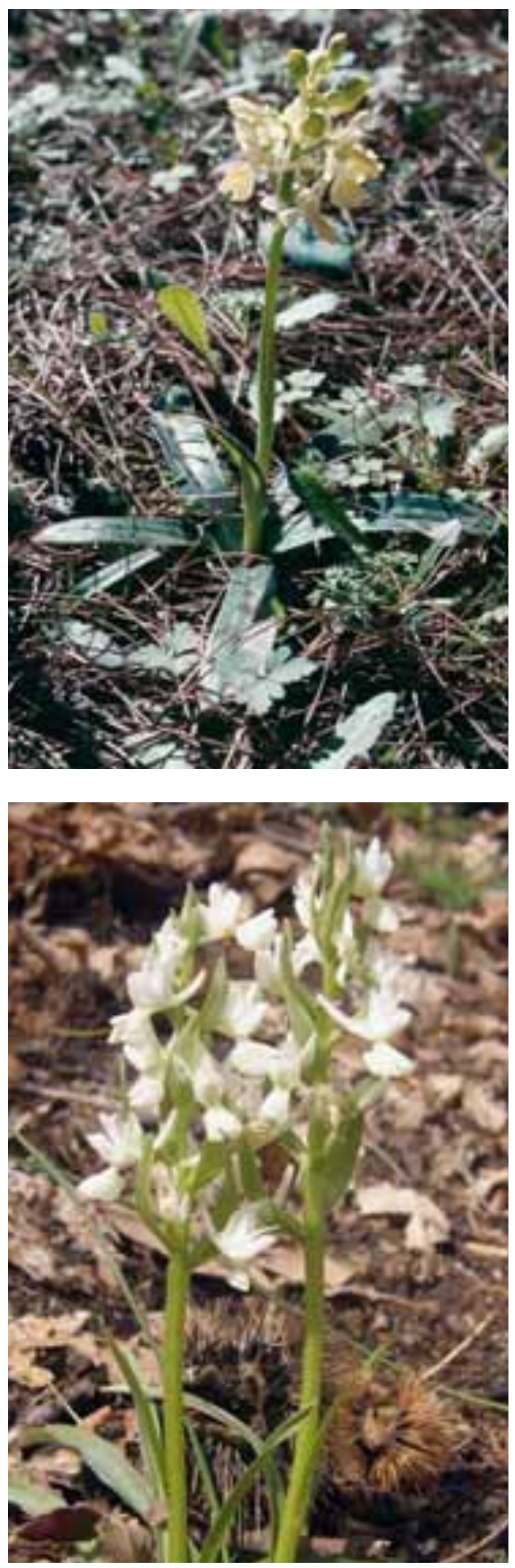

Mittelteil der Lippe dottergelb gefärbt mit braunen Punkten. Die Lippe wird gerade vorgestreckt und ist an den Rändern gezähnelt. Auch ihr Sporn ist aufwärts gebogen, aber die Blätter sind ungefleckt. In Nordwest-Tunesien und Nordost-Algerien ist die gelbblütige Orchis laeta (Algerisches Knabenkraut) verbreitet. Hier ist der Sporn aufsteigend und doppelt so lang wie der Fruchtknoten (Baumann 2006).

\section{Literatur}

Aeschimann, D., Lauber, K., Moser, D. M. \& Theurillat. J.-P. 2004: Flora Alpina. Bd. 2. - Bern. Baumann, H. 2005: Orchis pallens. In: AHO (Hrsg.): Die Orchideen Deutschlands. - Uhlstädt-Kirchhasel. Baumann, H. \& Künkele, S. 1988: Die Orchideen Europas. - Stuttgart.

Baumann , H., Künkele, S. \& Lorenz, R. 2006:

Die Orchideen Europas. - Stuttgart.

Buttler, K. P. 1986: Orchideen. - München.

Eberle, G. 1982: Die Orchideen der deutschen Heimat. 4. Aufl. - Frankfurt.

FülleR, F. 1962: Orchis und Dactylorchis.

Neue Brehm-Bücherei 286. - Wittenberg.

Kretzschmar, H. 2008: Die Orchideen Deutschlands und angrenzender Länder. - Wiebelsheim.

Künkele, S. \& Baumann, H.: Orchidaceae.

In: Sebald, O., Seybold, S. \& Philippi, G. 1998 (Hrsg.): Die Farn- und Blütenpflanzen Baden-Württembergs.

Bd.8. - Stuttgart.

Presser, H. 2002: Orchideen. 2. Aufl. - Hamburg. Reinhardt, H. R., Gölz, P., Peter, R. \& Wildermuth, H. 1991: Die Orchideen der Schweiz und angrenzender Gebiete. - Egg.

Seybold, S. 1986: Die Orchideen des Leonhart Fuchs. - Tübingen.

Vӧтн, W. 1999: Lebensgeschichte und Bestäuber der Orchideen am Beispiel Niederösterreichs. - Stapfia 65: $1-257$.

Abb. 9 (oben): Orchis provincialis.

Abb. 10 (unten): Dactylorhiza romana, eine blassgelbe Orchidee aus dem Mittelmeerraum, hier am Ätna auf Sizilien. 